\title{
Nurses' Sleep Quality of "Fangcang" Hospital in China during the COVID-19 Pandemic
}

\author{
Long Huang ${ }^{1,2}$ (D) Wansheng Lei ${ }^{1} \cdot$ Huan $\mathrm{Liu}^{3} \cdot$ Ronghua Hang $^{2} \cdot \mathrm{Xiubin}^{\mathrm{TaO}^{3}} \cdot$ \\ Yuxin Zhan ${ }^{4}$
}

Accepted: 19 September 2020 / Published online: 26 October 2020

(C) Springer Science+Business Media, LLC, part of Springer Nature 2020

Due to the high infectivity and long incubation period of new coronary pneumonia virus (Li et al. 2020; Rothe et al. 2020), COVID-19 has evolved into a global pandemic, and more cases and isolation measures appear in the news. Since China first reported COVID-19 in December 2019, as of May 11, 2020, a total of 234 countries and regions have reported 234,073 confirmed cases and 9840 deaths. Although various measures have been taken globally to combat new coronary pneumonia for more than 5 months, the number of confirmed cases and deaths worldwide is still increasing dramatically. COVID-19 poses a huge challenge to public health worldwide (Pan et al. 2020) and has caused serious and widespread negative effects on human beings (Lin 2020; Ahorsu et al. 2020). The uncertainty and low predictability of COVID-19 not only threaten people's physical health, but also affect people's mental health, especially emotional, stress, and sleep problems (Rothe et al. 2020)

COVID-19 is mainly spread through droplets, aerosols, etc. Therefore, the closer you are to the diagnosed patient, the more likely people are to be infected. People are required to stop work, suspend school, stop production, and restrict travel, so as to reduce the chance of exposure to COVID-19 patients. However, this isolation will also have a negative impact on people's mental health. But relative to COVID-19's direct physical and mental harm, isolation is still a good choice. But what about nurses on the frontline? Nurses who test and treat patients with COVID-19 have a

Long Huang and Wansheng Lei contributed equally to this work.

Huan Liu

wnyxyliuhuan@foxmail.com

$\triangle$ Ronghua Hang

344715850@qq.com

1 School of Psychology, Jiangxi Normal University, Nanchang, People's Republic of China

2 School of Humanities and Management, Wannan Medical College, Wuhu, People's Republic of China

3 Department of Nursing, The First Affiliated Hospital of Wannan Medical College, Wuhu, People's Republic of China

4 Department of Nursing, Union Hospital, Tongji Medical College, Huazhong University of Science and Technology, Wuhan, People's Republic of China 
higher risk of exposure than the general public and bear a huge mental burden. During COVID-19, nurses and other medical staff were mainly affected by the following aspects. First, COVID-19 is highly contagious. Many of the earliest medical workers in China who were exposed to COVID-19 were infected with COVID-19. It is reported that approximately 3000 medical personnel in China are infected with COVID-19, 40\% of which are infected in hospitals. At least 2629 health workers are infected in Italy, accounting for $8.3 \%$ of the total cases (Aljazeera 2020). Other countries have also reported many cases of nosocomial infections. The increasing number of cases increases the pressure and worries of medical staff (Lai et al. 2020; Adams and Walls 2020; Kushal et al. 2018; Chan-Yeung 2004), bringing them great mental pressure. They will worry about whether they will be infected and whether they will continue to infect their colleagues and family members. Second is the heavy workload. In an increasingly burdened medical system, excessive stress can cause job burnout. Work-related stress, including multiple clinical activities, depression in the face of countless deaths, prolonged work shifts, and the most diverse unknown factors, is a potential cause of concern for health professionals. Heavy workload is an important indicator of mental fatigue (Adams and Walls 2020; Kushal et al. 2018). Weekly long hours of work during COVID-19 increase new stress (Huang et al. 2020; Eddy et al. 2020; Wu et al. 2020), which may be related to fear of infection and excessive physical exertion. If you cannot relax for a long time, the nurse is easily burnout (Liu and Aungsuroch 2019). Nurse burnout is closely related to the physical and mental health of nurses. In addition, the relationship between the nurse and the patient, and the social support of family and friends are also important factors that affect the nurse's mental health. Social support has been found to relieve individual psychological problems (Su et al., 2015). Therefore, the increase in the number of diagnosed and suspected cases, the increase in workload, the consumption of personal protective equipment, the patient's mistrust, and the lack of social support may increase the psychological burden of nurses, causing problems such as anxiety, depression, and sleep disorders (The State Council of China 2020, Lai et al. 2020, Lee et al. 2007)

Previous studies have investigated the sleep status and influencing factors of firstline nurses during COVID-19. It was found that the sleep status of first-line medical staff was affected by COVID-19 to varying degrees. The level of social support of medical staff is significantly related to sleep quality, and stress level has a negative impact on sleep quality (Xiao, Zhang, Kong, Li, and Yang). However, as far as we know, no studies have investigated the sleep quality of nurses in "Fangcang" hospitals during the COVID-19 epidemic. "Fangcang" hospital is a new "cabin of life" for the centralized treatment of mild patients with COVID-19. Due to the limited capacity of specialized hospitals during the outbreak of the COVID-19, China took the lead in adopting "Fangcang" hospitals to treat mild patients. A total of 15 "Fangcang" hospitals have been put into use in China and have treated 12,000 mild patients. With China's creative use of large-scale shelter hospitals to prevent and control the epidemic, good results have been achieved. Russia, Britain, Italy, Iran, Serbia, and other countries have learned from Chinese experience and established "Fangcang" hospitals. The nurses in the "Fangcang" hospital have a new role. There may be differences in their sleep status and stress levels with other first-line nurses. Therefore, our research attempts to explore the sleep quality and its influencing factors of nurses in Chinese "Fangcang" hospitals, in order to provide support for the intervention on the sleep quality of nurses in "Fangcang" hospitals around the world. 


\section{Objective and Methods}

\section{Study Population and Sample}

The nurses of 15 "Fangcang" hospitals were selected as the participants of the survey in Wuhan, China. The snowball sampling method was used to invite subjects. An initial set of invitees (30 participants) was chosen to ensure a broad representation of age, gender, and hospital. This group of invitees forwarded the questionnaire to ten of their colleagues or friends who they deemed suitable for the survey, and the second group forwarded the questionnaire in the same way. A total of 966 valid questionnaires were received through online surveys. There were 85 males and 881 females; the average age was $31.92 \pm 5.89$ years old. The questionnaires were anonymous to ensure the confidentiality and reliability of data.

\section{Rating Instruments}

The demographic characteristic questionnaire includes content such as gender, age, working years, technical titles, the original department, and the number of patients. In addition, we used a 4-point scoring system to focus on investigating the trust of patients (Do you think the patients trust you in the "Fangcang" hospital?) and the support of family (Does your family support you in supporting Wuhan against COVID-19?) in nurse's self-evaluation: $0=$ distrust/ nonsupport, $1=$ low trust/low support, $2=$ medium trust/medium support, $3=$ high trust $/$ high support. In this study, no nurses chose "distrust and nonsupport".

The sleep quality of nurses was measured by using the Pittsburgh Sleep Quality Index (PSQI; Buysse et al. 1989), and the international guidelines regarding cross-cultural adaptation were used to translate the PSQI into Chinese. The scale was used to measure sleep quality and sleep disturbances in the past month, which comprised seven dimensions consisting of 19 items, including sleep quality, sleep duration, sleep latency, sleep efficiency, sleep disturbances, use of sleep medications, and daytime dysfunction. Each dimension was scored from 0 to 3 , and the total score, which was the sum of the scores from each dimension, ranged from 0 to 20. A higher score indicated lower sleep quality (Carpenter and Andrykowski 1998). In this study, Cronbach's alpha for internal consistency of the scale was 0.83 .

Stress was measured by using the Perceived Stress Scale, Chinese version (CPSS) (Yang et al. 2009; Cohen et al. 1983), which has been used extensively in prior research on stress in Chinese populations (Yang and Huang 2003). This scale comprised 14 items that addressed perceptions of stress during the month prior to the survey, which includes two dimensions of loss of control and tension, a total of 14 items. Each dimension was scored from 1 to 5, and the total score, which was the sum of the scores from each dimension, ranged from 14 to 70 . A higher score indicated higher stress perceived. In this study, Cronbach's alpha for internal consistency of the scale was 0.82 .

\section{Data Analysis}

All the statistical analyses were performed with IBM SPSS Statistics 21.0, with a two-tailed probability value of $<0.05$ considered to be statistically significant. The $t$-test was used to test group differences of continuous variables. Correlation of continuous variables was detected using Pearson's correlation analysis. Hierarchical multiple regression was applied to examine sleep quality and influencing factors of nurses in "Fangcang" hospital. 


\section{Ethical Considerations}

The ethics committee of Wannan Medical College approved this study. All participants voluntarily gave their informed consent to participate in the study after being informed about the purpose of the study. The procedures of this study complied with the provisions of the Declaration of Helsinki regarding research on Human participants.

\section{Results}

\section{Sleep Quality of Nurses in "Fangcang" Hospital}

It was found that the average PSQI of nurses in "Fangcang" hospitals was 6.77. $40.6 \%$ of nurses had a PSQI score between 1 and $5,44.2 \%$ of nurses with $6-10$ points, $12.6 \%$ with $11-15$ points, and $2.6 \%$ with $16-20$ points. In general, the sleep quality of nurses in the "Fangcang" hospital is moderate. The results of a general demographic survey on the sleep quality of nurses in the "Fangcang" hospital are shown in Table 1. The study found that the sleep quality of nurses in "Fangcang" hospitals differed significantly in terms of working years $(F=5.21, p=0.001)$, technical titles $(F=6.25, p=0.002)$, number of patients $(F=4.68, p=0.003)$, family's support $(F=3.56, p=0.029)$, and patient trust $(F=7.33, p=0.001)$. Multiple comparison (LSD) found that the sleep quality of nurses who worked for more than 16 years was significantly worse than that of nurses who worked for 5-10 years and within 5 years $(p s<0.01)$. The sleep quality of supervisor nurse is significantly worse than that of nurse practitioner and licensed practical nurses $(p s<0.01)$. The sleep quality of nurses with high-level family's support is significantly better than that of nurses with low-level and medium-level family's support $(p s<0.05)$. The sleep quality of nurses with high-level patient's trust is significantly better than nurses with low-level and medium-level patient's trust $(p s<0.01)$. The sleep quality of nurses with $31-40$ and more than 40 patients was significantly worse than that of nurses with less than 30 patients $(p s<0.05)$. No significant differences were found in the sleep quality of nurses in terms of education, department, and gender ( $p$ s $>0.05)$.

\section{Correlations among Continuous Variables}

Correlation analysis of working years, patient trust, family's support, number of responsible patients, stress perception, and sleep quality. Pearson's correlation analysis showed that sleep disturbances were significantly positively correlated with working years, number of patients, and stress perception $(p s<0.01)$. Sleep disturbances were significantly negatively correlated with patient trust and family's support $(p s<0.01)$. Stress perception was significantly negatively correlated with patient's trust and family's support $(p s<0.001)$, and stress perception was significantly positively correlated with gender $(p s<0.01)$. See Table 2 .

\section{Hierarchical Regression Analyses}

Using the method of stepwise regression analysis, we discussed the prediction of PSQI score by gender, working years, number of patients, patient's trust, family's 
Table 1 Demographic characteristics of the study subjects $(N=966)$ and univariate analysis for the factors related to the level of sleep quality

\begin{tabular}{|c|c|c|c|c|c|}
\hline \multirow[t]{2}{*}{ Variable } & & \multirow{2}{*}{$\begin{array}{l}\text { Number of subjects } \\
n(\%)\end{array}$} & \multicolumn{3}{|l|}{ PSQI } \\
\hline & & & Mean $\pm \mathrm{SD}$ & $F / t$ & $p$ \\
\hline \multirow[t]{4}{*}{ Working years } & $<5$ & $206(21.3 \%)$ & $6.16 \pm 3.43$ & 5.208 & .001 \\
\hline & $6-10$ & $352(36.4 \%)$ & $6.57 \pm 3.60$ & & \\
\hline & $11-15$ & $221(22.8 \%)$ & $7.00 \pm 3.62$ & & \\
\hline & $>16$ & $187(19.5 \%)$ & $7.52 \pm 4.00$ & & \\
\hline \multirow[t]{2}{*}{ Education background } & Junior college or below & $230(23.8 \%)$ & $6.50 \pm 3.57$ & -1.237 & .216 \\
\hline & College or above & $736(76.2 \%)$ & $6.85 \pm 3.71$ & & \\
\hline \multirow[t]{2}{*}{ Original department } & Infection-related departments & $341(35.3 \%)$ & $6.56 \pm 3.44$ & -1.287 & .198 \\
\hline & Others & $625(64.7 \%)$ & $6.88 \pm 3.79$ & & \\
\hline \multirow[t]{3}{*}{ Technical titles } & Licensed practical nurses & $137(14.2 \%)$ & $6.09 \pm 3.35$ & 6.251 & .002 \\
\hline & Nurse practitioner & $458(47.4 \%)$ & $6.57 \pm 3.68$ & & \\
\hline & Supervisor nurse & $371(38.4 \%)$ & $7.25 \pm 3.73$ & & \\
\hline \multirow[t]{2}{*}{ Gender } & Male & $85(8.7 \%)$ & $6.34 \pm 3.73$ & -1.116 & .265 \\
\hline & Female & $881(91.3 \%)$ & $6.81 \pm 3.67$ & & \\
\hline \multirow[t]{3}{*}{ Support degree } & Low & $59(6.1 \%)$ & $7.56 \pm 3.80$ & 3.556 & .029 \\
\hline & Medium & $349(36.1 \%)$ & $7.03 \pm 3.65$ & & \\
\hline & High & $558(57.8 \%)$ & $6.52 \pm 3.66$ & & \\
\hline \multirow[t]{3}{*}{ Trust degree } & Low & $49(5.1 \%)$ & $7.92 \pm 4.69$ & 7.326 & .001 \\
\hline & Medium & $535(55.4 \%)$ & $7.02 \pm 3.68$ & & \\
\hline & High & $382(39.5 \%)$ & $6.26 \pm 3.45$ & & \\
\hline \multirow[t]{4}{*}{ Number of patients } & $<20$ & $230(23.8 \%)$ & $6.36 \pm 3.53$ & 4.680 & .003 \\
\hline & $21-30$ & $338(35.0 \%)$ & $6.43 \pm 3.40$ & & \\
\hline & $31-40$ & $153(15.8 \%)$ & $7.29 \pm 3.79$ & & \\
\hline & $>40$ & $245(25.4 \%)$ & $7.29 \pm 4.00$ & & \\
\hline
\end{tabular}

support, and stress perception, see Table 3. The results found that working years and the number of patients positively predicted the nurse's PSQI score, and patient's trust can significantly negatively predict the nurse's PSQI score. Family's support has no predictive effect on the nurse's PSQI score. After adding the variable of stress perception, it was found that stress perception has a significant positive predictive effect on the nurse's PSQI score. At this time, the predictive effect of patient's trust on the nurse's PSQI score disappears. This shows that stress perception may be an intermediary factor between patient's trust and sleep quality. Further stratified regression analysis of pressure perception is shown in Table 4. It was found that both patient's trust and family's support negatively predicted nurses' pressure perception. This indicates that stress perception acts as a complete mediator between patient's trust and sleep quality.

Table 2 Correlations among sleep quality, stress perception, and related variables

\begin{tabular}{|c|c|c|c|c|c|c|c|}
\hline Variable & Mean \pm SD & 1 & 2 & 3 & 4 & 5 & 6 \\
\hline 1 Working years & $2.40 \pm 1.03$ & 1 & & & & & \\
\hline 2 Trust degree & $2.34 \pm 0.57$ & 0.063 & 1 & & & & \\
\hline 3 Support degree & $2.52 \pm 0.61$ & $0.064^{*}$ & $0.285^{* * *}$ & 1 & & & \\
\hline 4 Number of patients & $3.43 \pm 1.11$ & 0.012 & -0.038 & -0.003 & 1 & & \\
\hline 5 CPSS & $33.49 \pm 7.82$ & -0.062 & $-0.274^{* * *}$ & $-0.228^{* * *}$ & 0.039 & 1 & \\
\hline 6 PSQI & $6.77 \pm 3.68$ & $0.120^{* * * *}$ & $-0.123^{* * *}$ & $-0.087^{* *}$ & $0.110^{* *}$ & $0.348^{* * * *}$ & 1 \\
\hline
\end{tabular}

Note: ${ }^{*} p<0.05, * * p<0.01, * * * p<0.001$ 
Table 3 Hierarchical multiple regression results of sleep quality

\begin{tabular}{|c|c|c|c|c|c|c|}
\hline & \multicolumn{2}{|c|}{ Equation 1 (variable: PSQI) } & \multicolumn{2}{|c|}{ Equation 2 (variable: PSQI) } & \multicolumn{2}{|c|}{ Equation 3 (variable: PSQI) } \\
\hline & $\beta$ & $t$ & $\beta$ & $t$ & $\beta$ & $t$ \\
\hline Gender & 0.009 & 0.270 & 0.002 & 0.072 & -0.030 & -0.993 \\
\hline Working years & 0.124 & $3.796^{* * *}$ & 0.134 & $4.140^{* * *}$ & 0.151 & $4.946^{* * *}$ \\
\hline Number of patients & & & 0.101 & $3.196^{* *}$ & 0.092 & $3.082^{* *}$ \\
\hline Support degree & & & -0.062 & -1.893 & -0.006 & -0.201 \\
\hline Trust degree & & & -0.108 & $-3.261^{* * *}$ & -0.033 & -1.038 \\
\hline CPSS & & & & & 0.346 & $10.988^{* * * *}$ \\
\hline$R^{2}$ & $0.016^{* * * *}$ & & $0.047^{* * *}$ & & $0.153^{* * * *}$ & \\
\hline$\Delta R^{2}$ & $0.016^{* * *}$ & & $0.031^{* * * *}$ & & $0.107^{* * * *}$ & \\
\hline$F$ & $7.837^{* * * *}$ & & $9.394^{* * * *}$ & & $28.926^{* * *}$ & \\
\hline
\end{tabular}

Note: $* p<0.05, * * p<0.01, * * * p<0.001$

\section{Discussion}

\section{Current Status of Sleep Quality of Nurses in "Fangcang" Hospital}

Frontline medical staff, especially those in Wuhan, have close contact with COVID-19infected persons. It is often reported that they are subjected to excessive workload, isolation, and discrimination (Kang et al. 2020). Therefore, they are prone to physical fatigue, fear, emotional disorders, and sleep problems. A recent study involving 1563 health professionals found that $36.1 \%$ reported sleep disorders (Liu et al. 2020). However, our research found that the sleep quality of nurses in the "Fangcang" hospital was better than that of the study. This study found that the average PSQI score of nurses at the "Fangcang" hospital in the most severely affected area in China was 6.77 . Nurses with poor and very poor sleep quality only accounted for $12.6 \%$ and $2.6 \%$, respectively. This shows that the sleep quality of nurses in the "Fangcang" hospital is generally in a moderate state. This is contrary to the initial prediction of the "Fangcang" hospital by ordinary people. In the early days of the treatment of COVID-19 patients in the "Fangcang" hospital, some people had a high degree of distrust in the "Fangcang" hospital, even thinking that this was a "refugee camp" and "concentration camp". In fact, the living environment of "Fangcang" hospital is far beyond people's imagination. Unlike medical staff in the intensive care unit who are under tremendous pressure to face death

Table 4 Hierarchical multiple regression results of stress perception

\begin{tabular}{|c|c|c|c|c|}
\hline & \multicolumn{2}{|c|}{ Equation 1 (variable: CPSS) } & \multicolumn{2}{|c|}{ Equation 2 (variable: CPSS) } \\
\hline & $\beta$ & $t$ & $\beta$ & $t$ \\
\hline Gender & 0.109 & $3.311 * *$ & 0.095 & $3.031 * *$ \\
\hline Working years & -0.076 & $-2.330^{*}$ & -0.050 & -1.583 \\
\hline Number of patients & & & 0.026 & 0.865 \\
\hline Support degree & & & -0.162 & $-5.088^{* * *}$ \\
\hline Trust degree & & & -0.216 & $-6.756^{* * *}$ \\
\hline$R^{2}$ & $0.012^{* *}$ & & $0.103^{* * *}$ & \\
\hline$\Delta R^{2}$ & $0.014^{* *}$ & & $0.094^{* * *}$ & \\
\hline$F$ & $6.840^{* *}$ & & $23.188^{* * * *}$ & \\
\hline
\end{tabular}

Note: ${ }^{*} p<0.05, * * p<0.01, * * * p<0.001$ 
(Lima et al. 2020; Carvalho et al. 2020; Moreira and Rolim Neto 2020; Reuters 2020). "Fangcang" hospital received mild patients of COVID-19, almost no deaths, and many patients were successfully discharged. Therefore, the internal environment of the "Fangcang" hospital is relaxed, peaceful, and free and lively. Square dance, drama, reading, and learning can be seen everywhere. Compared with the heavily guarded environment outside the "Fangcang" hospital, the "Fangcang" hospital is more like a paradise. In addition, although more than 3000 medical workers in Hubei Province were infected at the beginning of COVID19, medical staff supporting Wuhan from other provinces and cities in China have always maintained a "zero infection" (Beijing Daily 2020). This brings a great sense of security to nurses in "Fangcang" hospitals from various provinces and cities in China. In addition, a good atmosphere and sleep quality may also be related to the nurse's high sense of responsibility and preparation. The nurses in the "Fangcang" hospital actively requested to participate in support and treatment, and received support and encouragement from their loved ones (there were no cases in which the nurse's family did not support this study). Bruce Aylward said that Chinese medical workers have demonstrated their sense of responsibility and collective action in the fight against COVID-19. They all have a mentality of working hard to complete the task (Kupferschmidt and Cohen 2020). Therefore, the sleep quality of nurses is less affected by the epidemic in the "Fangcang" hospital.

\section{Influencing Factors of Sleep Quality of Nurses in "Fangcang" Hospital}

The results of this study show that with the increase of working years, the sleep quality of nurses is declining. This may be related to the general law of the human body. With the increase of age, people's demand for sleep quality generally decreases, and sleep quality also gradually declines (Lemola and Richter 2013). The relationship between technical titles and sleep quality is inversely related, which is the same as the existing research results (Yu et al. 2018). On the one hand, it may be that technical titles often have a higher relationship with working years. In the evaluation of technical titles in Chinese hospitals, there are strict year requirements for the promotion of each level of titles. On the other hand, it may be because nurses with high technical titles and long working years assume more important responsibilities in the "Fangcang" hospital. They are the leaders of their respective teams and are directly responsible for the treatment and care of patients.

In addition, the number of patients under the care of the nurse has a strong predictive effect on sleep quality. The higher the number of patients, the worse the sleep quality. During the outbreak of the COVID-19 epidemic, although hundreds of medical teams were sent from various provinces and cities in China to support Hubei, they still faced huge workload. Our study found that $40 \%$ of nurses in the "Fangcang" hospital need to be responsible for more than 30 patients. The sleep quality of this part of nurses has been seriously affected, which is significantly worse than that of nurses with fewer than 30 patients. When nurses care for COVID-19 patients, there is a high risk of occupational exposure. The greater the number of patients, the longer the working hours, which increases the possibility of infection and excessive physical exertion, resulting in greater stress (Huang et al. 2020; Eddy et al., 2020; Wu, 2020) and burnout (Liu and Aungsuroch 2019), which affects sleep quality. Therefore, on the frontline of the COVID-19 epidemic, nursing managers should strengthen the allocation and management of human resources, arrange shifts scientifically, reduce the work intensity of nurses, and thus improve the quality of sleep of nurses. 
Social support is an important protective factor for psychological resilience, which can alleviate psychological pressure and relieve psychological barriers (Su and Guo 2015). Our research found that the family's support has a positive effect on nurses' sleep quality and can also reduce nurses' stress perception. This is consistent with existing research results (Xiao et al. 2020). In our study, the nurses in the "Fangcang" hospital were all medical staff from hospitals in various provinces and cities in China. They are far from their hometowns, risking themselves and their family members being infected, and heading to Wuhan to fight the epidemic. This process requires the support of their families. Moreover, because the COVID19 virus is different from the daily disease care, which has a strong ability to spread, nurses must stay alone in the room after completing the work in order to reduce cross-infection (National Health Commission of the People's Republic of China, 2020). Isolation measures may lead to boredom, loneliness, anxiety, and other emotions (Brooks et al. 2020). The nurses in the "Fangcang" hospitals need more social support than ever, especially family support. Our research also confirms this. Nurses who received more family support had significantly less stress perception and better sleep quality than nurses who received less family support. Social support can help reduce stress and improve sleep by reducing the physiological response and inappropriate behavior that may be caused by the perception of threats to stress events. Therefore, nursing managers should help nurses actively mobilize their social support system to solve their family worries. China has established many social support systems. For example, children of nurses can get extra points in the entrance examination, increasing financial incentives, and improving their social status. These measures have given great support to the nurses in "Fangcang" hospital, which may also be an important reason for the moderate sleep quality of the nurses in the "Fangcang" hospital found in this study.

Patients in the "Fangcang" hospital have a high degree of trust in nurses, and no nurse thinks that some patients do not trust them. This may be because the public welfare of the "Fangcang" hospital determines the basic reason for the harmonious nurse-patient relationship in the "Fangcang" hospital. From the perspective of patients, since they no longer worry that medical staff will use their diseases to make money, the trust in nurses is greatly enhanced, and the mutual suspicion between nurses and patients has disappeared. At the same time, the patient will also be in a grateful mood, try his best to overcome the difficulties, and try not to "trouble" the medical staff. From the perspective of medical staff, the trust, respect, and friendliness of patients greatly enhance their professional pride and honor, so they will treat patients more friendly and patiently. As a result, the nurse-patient relationship has entered a process of benign interaction (Guo 2020). This makes the patient more trustful, so the overall quality of sleep is better. It can be inferred from this that public welfare is an important measure to resolve the contradiction between nurses and patients. Moreover, our study found that patient trust is a predictor of sleep quality and stress perception, and stress perception is an intermediary factor between patient's trust and sleep quality. The higher the patient's trust, the less pressure the nurse perceives, which in turn causes the nurse to maintain a good sleep. Studies have found that work stress is related to a series of physical and mental health problems (e.g., insomnia and anxiety) (Nakao 2010). According to the research of "Fangcang" hospital, it can be found that a good nurse-patient relationship is a key factor in maintaining nurses' sleep quality and stress perception. The important thing to build a good nurse-patient relationship is to get rid of the shackles of capital and return the nurse-patient relationship to pure relief and be rescued. 


\section{Conclusions}

In general, the sleep quality of nurses in "Fangcang" hospitals is at a good level. The number of patients, family support, the trust of patients, and stress perception are the most important factors affecting sleep quality. Therefore, reducing the number of patients under the care of nurses and improving the relationship between nurses and patients is an important way to improve stress perception and the sleep quality of nurses in "Fangcang" hospitals. Our research results can provide reference for the managers of "Fangcang" hospitals in other countries. Nursing managers in "Fangcang" hospitals should strengthen the construction of the nurses' support system, arrange nurses' work burden scientifically and reasonably, and maintain good nurse-patient relationship, thereby reducing nurses' psychological pressure and improving nurses' sleep quality in "Fangcang" hospital.

Acknowledgements This study was supported by MOE (Ministry of Education in China) Project of Humanities and Social Sciences (Grants Nos. 20YJC190006), the Outstanding Young Talent Support Program Project of Anhui Province (Grants Nos. gxyq2020160), the prevention and control science and technology emergency project for coronavirus disease 2019 of Wuhu (Grants Nos. 2020rkx1-5), the Philosophy and Social Science Planning Project of Anhui Provincial (Grants Nos. AHSKQ2019D059), and the University Science Research Project of Anhui Provincial Department of Education (Grants Nos. SK2018A0192).

\section{Compliance with Ethical Standards}

Conflict of Interest The authors declare that they have no conflicts of interest.

Informed Consent Informed consent was obtained from all individual participants included in the study.

\section{References}

Adams, J. G., \& Walls, R. M. (2020). Supporting the health care workforce during the COVID-19 global epidemic. JAMA., 323, 1439-1440. https://doi.org/10.1001/jama.2020.3972.

Ahorsu, D. K., Lin, C. Y., Imani, V., Saffari, M., Griffiths, M. D., \& Pakpour, A. H. (2020). The fear of COVID19 scale: Development and initial validation. International journal of mental health and addiction. https://doi.org/10.1007/s11469-020-00270-8.

Aljazeera (2020). Thousands of medical staff infected with coronavirus in Italy. https://www.aljazeera. com/news/2020/03/rising-number-medical-staff-infected-coronavirus-italy-200318183939314.html.

Beijing Daily (2020). National support Wuhan medical staff "zero infection" new crown pneumonia[EB/OL]. https://ie.bjd.com.cn/5b165687a010550e5ddc0e6a/contentApp/5b16573ae4b02a9fe2d558f9/AP5e620 dcbe4b00ca727d766ac?isshare $=1$ andapp $=9$ ca3764ea5f70b78andcontentType $=0$ andisBjh $=0$.

Brooks, S. K., Webster, R. K., Smith, L. E., Woodland, L., Wessely, S., Greenberg, N., \& Rubin, G. J. (2020). The psychological impact of quarantine and how to reduce it: Rapid review of the evidence. The Lancet., 395, 912-920. https://doi.org/10.1016/S0140-6736(20)30460-8.

Buysse, D. J., Reynolds, C. F., Monk, T. H., Berman, S. R., \& Kupfer, D. J. (1989). The Pittsburgh sleep quality index: A new instrument for psychiatric practice and research. Psychiatry Research, 28(2), 193-213. https://doi.org/10.1016/0165-1781(89)90047-4.

Carpenter, J. S., \& Andrykowski, M. A. (1998). Psychometric evaluation of the Pittsburgh Sleep Quality Index. Journal of Psychosomatic Research, 45, 5-13. https://doi.org/10.1016/S0022-3999(97)00298-5.

Carvalho, P. M. M., Moreira, M. M., Oliveira, M. N. A., Landim, J. M. M., \& Rolim Neto, M. L. (2020). The psychiatric impact of the novel coronavirus outbreak. Psychiatry Research, 286, 112902. https://doi. org/10.1016/j.psychres.2020.112902.

Chan-Yeung, M. (2004). Severe acute respiratory syndrome (SARS) and healthcare workers. International Journal of Occupational and Environmental Health, 10, 421-427. https://doi.org/10.1179 /oeh.2004.10.4.421. 
Cohen, S., Kamarck, T., \& Mermelstein, R. (1983). A global measure of perceived stress. Journal of Health and Social Behavior, 24, 385-396. https://doi.org/10.2307/2136404.

Guo, S. M. (2020). The shelter hospital is like a sociological experiment, revealing the answer to the contradiction between doctors and patients. Observer. https://www.guancha.cn/GuoSongMin/2020_02_28_538890. shtml?s=wapzwyzzwzbt

Huang, C., Wang, Y., Li, X., Ren, L., Zhao, J., Hu, Y., Zhang, L., Fan, G., Xu, J., Gu, X., Cheng, Z., Yu, T., Xia, J., Wei, Y., Wu, W., Xie, X., Yin, W., Li, H., Liu, M., Xiao, Y., Gao, H., Guo, L., Xie, J., Wang, G., Jiang, R., Gao, Z., Jin, Q., Wang, J., \& Cao, B. (2020). Clinical features of patients infected with 2019 novel coronavirus in Wuhan, China. The Lancet, 395, 497-506. https://doi.org/10.1016/s0140-6736(20)30183-5.

Kang, L., Li, Y., Hu, S., Chen, M., Yang, C., Yang, B. X., Wang, Y., Hu, J., Lai, J., Ma, X., Chen, J., Guan, L., Wang, G., Ma, H., \& Liu, Z. (2020). The mental health of medical workers in Wuhan, China dealing with the 2019 novel coronavirus. The Lancet Psychiatry., 7, e14. https://doi.org/10.1016/s2215-0366(20)30047$\mathrm{x}$.

Kupferschmidt, K., \& Cohen, J. (2020). Can China's COVID-19 strategy work elsewhere? Science, 367(6482), 1061-1062. https://doi.org/10.1126/science.367.6482.1061.

Kushal, A., Gupta, S., Metha, M., \& Singh, M. M. (2018). Study of stress among health care professionals: A systemic review. Int J Res Foundation Hosp Healthcare Adm., 6, 6-11. https://doi.org/10.5005/jp-journals10035-1084.

Lai, J., Ma, S., Wang, Y., Cai, Z., Hu, J., Wei, N., Wu, J., du, H., Chen, T., Li, R., Tan, H., Kang, L., Yao, L., Huang, M., Wang, H., Wang, G., Liu, Z., \& Hu, S. (2020). Factors associated with mental health outcomes among health care workers exposed to coronavirus disease 2019. JAMA Network Open., 3(3), e203976. https://doi.org/10.1001/jamanetworkopen.2020.3976.

Lee, A. M., Wong, J. G., Mcalonan, G. M., Cheung, V., Cheung, C., Sham, P. C., et al. (2007). Stress and psychological distress among SARS survivors 1 year after the outbreak. The Canadian Journal of Psychiatry., 52(4), 233-240. https://doi.org/10.1177/070674370705200405.

Lemola, S., \& Richter, D. (2013). The course of subjective sleep quality in middle and old adulthood and its relation to physical health. Journals of Gerontology, Series B: Psychological Sciences and Social Sciences, 68(5), 721-729. https://doi.org/10.1093/geronb/gbs113.

Li, Q., Guan, X., Wu, P., Wang, X., Zhou, L., Tong, Y., Ren, R., Leung, K. S. M., Lau, E. H. Y., Wong, J. Y., Xing, X., Xiang, N., Wu, Y., Li, C., Chen, Q., Li, D., Liu, T., Zhao, J., Liu, M., Tu, W., Chen, C., Jin, L., Yang, R., Wang, Q., Zhou, S., Wang, R., Liu, H., Luo, Y., Liu, Y., Shao, G., Li, H., Tao, Z., Yang, Y., Deng, Z., Liu, B., Ma, Z., Zhang, Y., Shi, G., Lam, T. T. Y., Wu, J. T., Gao, G. F., Cowling, B. J., Yang, B., Leung, G. M., \& Feng, Z. (2020). Early transmission dynamics in Wuhan, China, ofnovel coronavirusinfected pneumonia. The new England Journal of Medicine., 382, 1199-1207. https://doi.org/10.1056 /NEJMoa2001316.

Lima, C. K. T., Carvalho, P. M. M., Lima, I. A., Nunes, J. V. A. O., Saraiva, J. S., Souza, R., et al. (2020). The emotional impact of coronavirus 2019-nCoV (new coronavirus disease). Psychiatry Research, 287, 112915. https://doi.org/10.1016/j.psychres.2020.112915.

Lin, C. Y. (2020). Social reaction toward the 2019 novel coronavirus (COVID-19). Social Health and Behavior, 3(1), 1. https://doi.org/10.4103/SHB.SHB_11_20.

Liu, Y., \& Aungsuroch, Y. (2019). Work stress, perceived social support, self-efficacy, and burnout among Chinese registered nurses. Journal of Nursing Management., 75, 3076-3083. https://doi.org/10.1111 /jonm. 12828.

Liu, S, Yang, L. L, Zhang, C. X., Xiang, Y. T., Liu, Z., Hu, S., et al. (2020). 2019 novel coronavirus: Online mental health services. Lancet Psychiatry.

Moreira, M. M., \& Rolim Neto, M. L. (2020). What does the future hold for doctors and suicide? Psychiatry Research, 285, 112832. https://doi.org/10.1016/j.psychres.2020.112832.

Nakao, M. (2010). Work-related stress and psychosomatic medicine. Biopsychosoc Med., 4(1), 4. https://doi. org/10.1186/1751-0759-4-4.

Pan, Y., Guan, H., Zhou, S., Wang, Y., Li, Q., Zhu, T., Hu, Q., \& Xia, L. (2020). Initial CT findings and temporal changes in patients with the novel coronavirus pneumonia (2019-nCoV): A study of 63 patients in Wuhan, China. European Radiology, 30, 3306-3309. https://doi.org/10.1007/s00330-020-06731-x.

Reuters (2020). Special report: "all is well". In Italy, triage and lies for virus patients. https://www.reuters. com/article/us-health-coronavirus-italy-ethics-speci/special-report-all-is-well-in-italy-triage-and-lies-forvirus-patients-idUSKBN2133KG.

Rothe, C., Schunk, M., Sothmann, P., Bretzel, G., Froeschl, G., Wallrauch, C., Zimmer, T., Thiel, V., Janke, C., Guggemos, W., Seilmaier, M., Drosten, C., Vollmar, P., Zwirglmaier, K., Zange, S., Wölfel, R., \& Hoelscher, M. (2020). Transmission of 2019-nCoV infection from an asymptomatic contact in Germany. The new England Journal of Medicine., 382, 970-971. https://doi.org/10.1056/NEJMc2001468. 
Su, Q., \& Guo, L. L. (2015). Relationship between psychological elasticity, work stress and social support of clinical female nurses. Chinese Occupational Medicine., 42(1), 55-58.

The State Council of China 2020. A notification to set up nationwide psychological assistance hotlines against the 2019-nCoV outbreak. http://www.gov.cn/xinwen/2020-02/02/content_5473937.htm.

Wu, J. J., Song, X., Chen, F., et al. (2020). Survey of sleep quality of clinical front-line nurses and its influencing factors in the fight against new coronavirus pneumonia. Nursing Research 1-5. http://kns.cnki. net/kcms/detail/14.1272.r.20200214.1136.004.html.

Xiao, H., Zhang, Y., Kong, D. S., Li, S. Y., \& Yang, N. X. (2020). The effects of social support on sleep quality of medical staff treating patients with coronavirus disease 2019 (COVID-19) in January and February 2020 in China. Med Sci Monit, 26, e923549. https://doi.org/10.12659/MSM.923921.

Yang, T. Z., \& Huang, H. T. (2003). An epidemiological study on stress among urban residents in social transition period. Chinese Epidemiology., 24, 760-764.

Yang, T., Rockett, I. R., Yang, X., \& Xu, X. (2009). Patterns and correlates of stressamong rural Chinese males: A four-region study. Public Health, 123, 694-698.

Yu, S. J., Cao, Y. J., \& Ma, D. D. (2018). A survey on night sleep quality and daytime tiredness among shift nurses in a tertiary teaching hospital. Chinese Journal of Industrial Hygiene and Occupational Diseases, $36(11), 855-858$.

Publisher's Note Springer Nature remains neutral with regard to jurisdictional claims in published maps and institutional affiliations. 